\title{
Linguistic gem or just another pidgin?
}

\author{
Talking Hands: What Sign Language \\ Reveals about the Mind \\ by Margalit Fox \\ Simon \& Schuster: 2007.368 pp. \$27
}

\section{Neil Smith}

Al-Sayyid Bedouin Sign Language (ABSL) is a system of communication, created some 70 years ago by ten deaf people living in a village in Israel. In her elegantly written book, Margalit Fox claims that ABSL is "a language that is free of the influence of other languages, signed or spoken", and uses it as the starting point for an inquiry into whether innate properties of the human mind could be reflected in the emergence of this 'new' language over three generations.

Fox intersperses the account of her investigation with a masterly and accessible overview of sign languages and research into them over the past half century. Although they share their linguistic properties, sign languages are independent of spoken languages. For example, American Sign Language is more closely related to French Sign Language than it is to British Sign Language, and is unrelated to spoken American English. They have phonology, expressed through the shape, location, movement and orientation of the hands, and typically exhibit a rich morphology - for example, the sign for 'give' can be modified to portray repeated giving, continuous giving or giving to many recipients.

Most sign languages have a complex system of verb agreement, allowing signers to keep track of who does what to whom, and to exploit word-order variation for rhetorical effect, rather than to encode grammatical relations such as subject and object. Their syntax allows the formation of questions, negations, conditionals and so on, and they have a compositional semantics. Whatever can be said in spoken language, deaf signers can convey in sign language.

The evidence for this comes from analyses of sign languages and their historical development, and from psycholinguistic and brain-imaging experiments. Parallels between the signed and the spoken in every domain are so close that it is hard to tell from a linguistic description which kind of language is being discussed. The genetically determined human faculty of language seems to be largely neutral between the two modalities.

There are differences. Signed languages are more iconic and allow for a degree of simultaneity not possible in spoken language. You can frequently tell, post hoc, why a sign has the shape it does, and you can sign more than one morpheme - the smallest meaningful

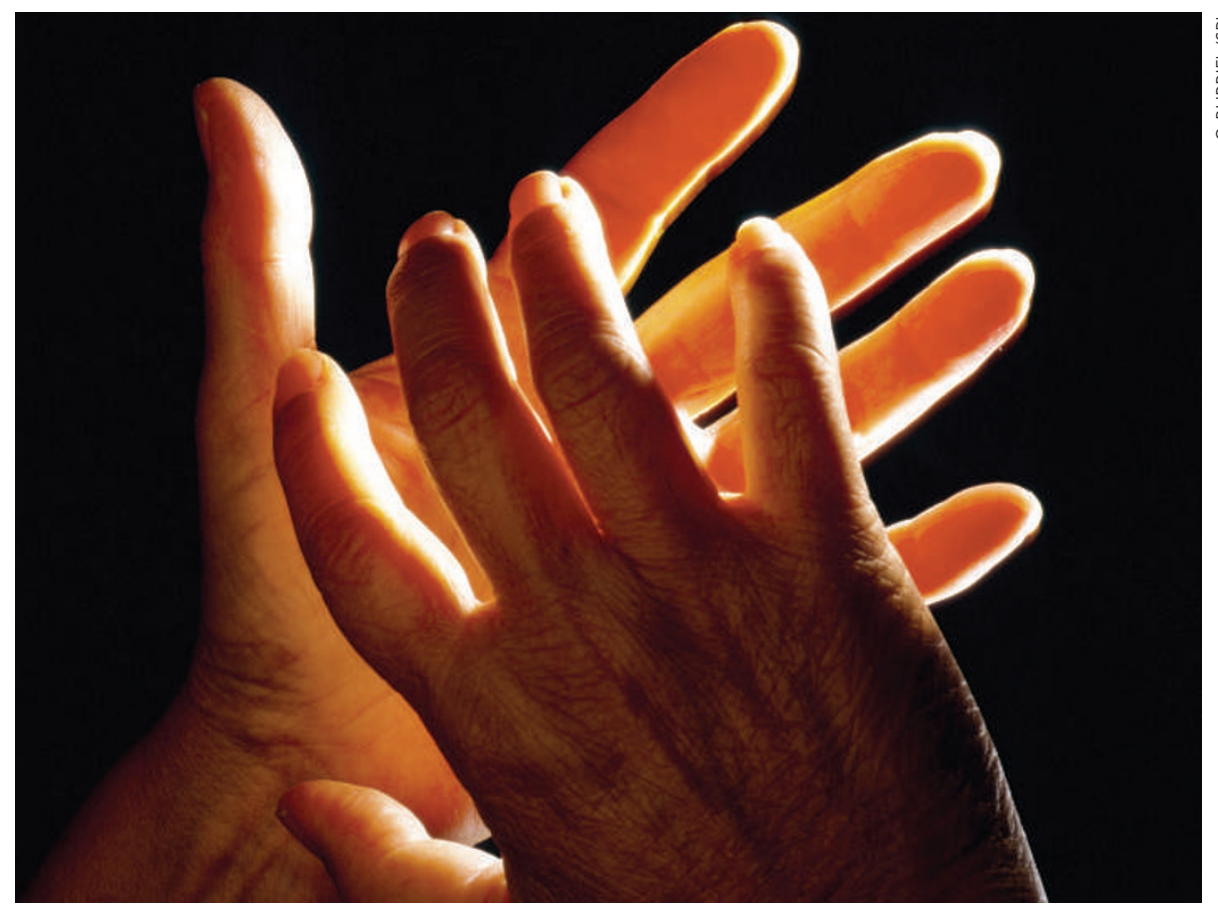

Can the emergence of a new sign language tell us anything about the innate properties of the mind?

linguistic unit - at a time. Yet deviations from iconicity are frequent and signed words, like spoken words, typically consist of linear sequences of 'locations' and 'movements'. The most striking difference lies in the importance of facial expression: in signed languages this can have many of the functions that grammar and intonation do in spoken languages.

Deaf people not exposed to sign languages may invent their own restricted system of communication, known as 'home-sign': a pidgin, which lacks many of the properties of a real language. In being passed on to succeeding generations, a pidgin may become a creole (the speaker's or signer's native language), and eventually turn into a full-fledged language. Pidgins have minimal morphology, creoles somewhat more, and full-fledged languages may be morphologically rich.

This progression, if it occurs without influence from other languages, is what Fox suggests can reveal innate properties of mind, and is what motivated the expedition to examine ABSL. But there are problems. Influence from other languages, both signed and spoken, has been considerable. Moreover, although pidgins are typologically identifiable, creoles are not linguistically well defined, and it is a fallacy that languages generally develop a rich morphology over time: some do (French), some do not (Chinese), and some lose much of their morphology (English).

Nevertheless, ABSL would indeed provide evidence for the operation of the human lan- guage faculty if it really had become "without doubt a fully functioning language". Suggestive evidence for the claim comes from the marking of grammatical relations, demonstrated syntactically by use of word order, or morphologically by use of agreement. Most sign languages rely on the latter, but ABSL chooses the former - a rigid subject-object-verb order.

Beyond this, the conclusions drawn by Fox and the team of four linguists she accompanied on the expedition are disappointing. ABSL has a large vocabulary but makes no use of spatial morphology and has no verb agreement, apparently because it is "too new". More surprisingly, "the language seemed to lack phonology" and "holistic words serve the communicative needs of their users admirably". So we are asked to believe that we have a 'language' with some (minimal) syntax but no morphology and, amazingly, no phonology.

Fox's conclusion that "spontaneously, naturally and with no outside influence, the deaf villagers created a new human language" is overstated. ABSL seems to be not so much a language, as a pidgin that is being creolized under the pervasive influence of Israeli Sign Language.

Neil Smith is professor emeritus of linguistics at University College London, Gower Street, London WC1E 6BT, UK.

\section{Correction}

In the Book review "The art of persuasion" (Nature 448, 751-752; 2007) the image erroneously portrayed Isaac Newton instead of Robert Boyle. 